\title{
Entre évaluation et gestion des risques, de l'importance de la précision du vocabulaire
}

\author{
Gérard Pascal \\ Directeur honoraire de recherches, INRA, 75007 Paris, France
}

L'article de Christian Ducrot, Mathilde Paul et Didier Calavas ${ }^{1}$ décrit parfaitement la suite d'événements qui a d'abord conduit au développement de l'«épidémie » d'ESB à partir du début des années 1980 en GrandeBretagne, puis dans beaucoup d'autres pays européens, et les mesures successives prises pour parvenir à la maîtrise progressive de la situation. Les difficultés d'application des mesures de gestion conduisant à des contaminations croisées sont clairement présentées et expliquent l'accumulation des décisions qui ont finalement abouti à l'interdiction totale des farines de viande et d'os (FVO) en élevage en 2000. La situation épidémiologique s'est aujourd'hui normalisée puisque seulement cinq cas (quatre par surveillance épidémiologique des animaux à risque et un par dépistage systématique à l'abattoir) ont été détectés en France en 2010 et trois seulement en 2011 chez des animaux à risque.

Le calme régnait donc sur le front de l'ESB, lorsque la Commission européenne a publié en juillet 2010 une feuille de route et a demandé l'avis des États membres de l'Union européenne (UE) sur la possibilité de lever une partie des interdictions alors en vigueur, en ce qui concernait l'usage en élevage de produits d'origine animale riches en protéines. Cette question constitue la seconde partie de l'article de Ducrot et al.

Dès octobre 2010, l'Agence nationale de sécurité sanitaire de l'alimentation, de l'environnement et du travail (Anses) et le Conseil national de l'alimentation (CNA, véritable parlement de l'alimentation) ont été saisis par les autorités françaises, la première sur les aspects sanitaires, le second sur les aspects socioéconomiques incluant l'acceptabilité sociale des mesures envisagées. Lors des

Auteur correspondant : gerard_pascal@yahoo.fr

${ }^{1}$ Cf. l'article de Ducrot et al. dans ce numéro «BSE risk and the use of meat and bone meal in the feed industry: perspectives in the context of relaxing control measures ». réunions du groupe de travail mis en place au sein du CNA pour traiter de la question, il est tout de suite apparu qu'une discussion ne serait possible qu'après avoir très clairement explicité les différences entre les farines de viande et d'os (FVO), responsables de la crise, et les produits dont la Commission envisageait la possible introduction en élevage avicole, porcin et piscicole, les protéines animales transformées (PAT). En effet, si ces deux types de produits étaient identiques, point n'était besoin d'examiner plus avant la question ; le refus s'imposait d'emblée. Il convenait de ne pas reproduire les erreurs du passé ! Après une clarification totale et une mise en évidence des différences essentielles entre FVO et PAT, le CNA a adopté, après plus d'un an de consultations et de discussions, un avis $\left(\mathrm{n}^{\circ} 70\right)$ rendu public le $1^{\mathrm{er}}$ décembre 2011 (accessible sur le site du CNA).

Immédiatement, malgré les précautions prises dans la rédaction de l'avis, la polémique a été déclenchée dans les médias, l'amalgame étant fait entre FVO et PAT. Le communiqué de presse de la présidence du CNA et sa réaction à un article du Monde du 5 décembre 2011 n'ont permis à cette polémique que de s'éteindre très progressivement, un avis du ministre de l'Agriculture selon lequel la France s'opposerait à toute modification des mesures en vigueur ayant largement contribué à la confusion.

Une bonne analyse de l'article de Ducrot et al. me semble devoir être facilitée par un exposé détaillé des différences entre l'utilisation passée de FVO et l'usage proposé d'emploi de PAT.

La crise de la vache folle est due à l'utilisation en élevage bovin - essentiellement chez les vaches laitières de farines de viande et d'os, constituées de cadavres d'animaux morts de différentes causes et de sousproduits d'abattoir renfermant - ce que l'on a ensuite qualifié de "matériaux à risques spécifiés » (MRS) - cervelle, moelle épinière, yeux, essentiellement, mais aussi colonne vertébrale ainsi que d'autres tissus et organes 
pouvant être concernés. La liste de ces MRS différait selon les analyses de risques, la liste française étant plus longue que celle de l'UE. Les FVO ont tout d'abord été interdites en élevage bovin, puis en élevage de ruminants. Des améliorations successives avaient été apportées à leur composition - élimination totale des cadavres, des MRS, traitement des FVO dans des conditions de température, temps et pression standardisées -, mais les difficultés de gestion et les jeux politiques, en particulier en France en période de cohabitation, ont conduit, comme évoqué plus haut, à l'interdiction totale des FVO en élevage. On peut d'ailleurs s'interroger sur l'opportunité de cette interdiction alors que de très nombreuses mesures de précaution avaient été prises. Les auteurs de l'article soulignent d'ailleurs combien il est difficile pour des épidémiologistes de juger des effets de l'interdiction d'utilisation des MRS en 1996 et du retrait total des FVO en 2000 sur l'évolution de la situation, alors que la maladie déclinait déjà très rapidement.

Les propositions de la Commission sont d'une tout autre nature. Il s'agit d'introduire en élevage, sous certaines conditions, des produits d'origine animale (porcs, volailles, poissons) riches en protéines dans les conditions suivantes :

- L'utilisation des PAT de ruminants est interdite en élevage.

- L'utilisation des PAT en élevage de ruminants est totalement interdite.

- Les matières premières doivent provenir d'animaux sains, déclarés propres à la consommation humaine.

- Les PAT issues de volailles seraient réservées à l'alimentation porcine ou aux poissons, celles de porcs à l'alimentation des volailles et des poissons. Le recyclage intra-espèce serait exclu.

Signalons que les PAT sont d'ores et déjà autorisées en alimentation des animaux de compagnie; ne peuton alors penser qu'elles ne présentent qu'un risque mineur, alors que l'on sait que le prion bovin peut relativement facilement franchir la barrière d'espèce chez le chat?
La question majeure qui se pose en matière d'évaluation de risques concernel'origine des dangers, c'est-à-dire la présence de prions bovins dans les PAT :

- Les prions se concentrent dans les MRS chez les bovins infectés ; ils n'ont jamais été mis en évidence dans le lait ou les muscles. Les MRS de ruminants sont séparés à l'abattoir et dénaturés avant destruction.

- Les prions bovins de même que ceux d'autres EST n'ont jamais été mis en évidence en conditions naturelles, chez les porcs, la volaille ou les poissons, mais seulement dans certains cas en conditions expérimentales extrêmes.

L'évaluateur de risques ne peut donc que conclure que le risque de la présence de prions bovins ou d'autres agents d'EST dans les aliments des porcs, volailles et poissons, du fait de l'introduction de PAT dans les conditions prévues par la Commission, est infime.

Des failles dans l'application des mesures prévues restent possibles et peuvent seules expliquer la présence d'un risque. Seul le gestionnaire du risque peut en apprécier la possibilité et décider de mesures de gestion destinées à le minimiser, en fonction de ses critères de mise en œuvre du « principe de précaution ». Les désaccords entre États, en particulier entre la France et l'UE, résultent le plus souvent non de désaccords entre scientifiques évaluateurs de risques, mais de différences dans le choix de ces critères.

L'information claire et transparente du citoyen impose d'exposer d'une part l'état de l'évaluation scientifique du risque par les collectifs experts, et d'autre part les critères de choix des modalités de gestion retenues par les décideurs administratifs ou politiques. Une séparation explicite entre les deux est nécessaire pour une bonne compréhension de la situation, en particulier dans l'exemple des farines animales et des protéines animales transformées, objet de l'article de Ducrot et al., afin que les critères retenus, autres que scientifiques, apparaissent comme justifiant des différences de décisions. L'avis du CNA, qui traite spécifiquement des aspects socioéconomiques, est très éclairant à ce sujet. 